\title{
EFFICIENT TECHNIQUE FOR AUTOMATIC EXTRACTION AND IDENTIFICATION OF ELEVATION FROM A REFERENCE IMAGE
}

\author{
Mohan P Pradhan \\ Department Of CSE, SMIT, SMU \\ mohanp_pradhan25@yahoo.com.sg \\ M K Ghose \\ Department Of CSE, SMIT, SMU \\ mkghose@smu.edu.in \\ Gourav Mittal \\ Department Of CSE, SMIT, SMU \\ gourav.mittal@live.com
}

\begin{abstract}
In Geographic information system (GIS) identification of elevation detail pertaining to a contour in a reference map or topological map plays an important role while creating digital elevation model or digital surface model of a terrain. Traditional technique involving manual identification of elevation demands greater effort and time where in one needs to tediously identify and enter the elevation value. These identified elevations can be later used for creating digital elevation model. In order to reduce the complexity involved in same, effective and efficient automated technique can be devised that identifies the elevation associated with a contour. In addition the automated technique should also ensure correctness and reliability of the elevation details identified. The alignment of elevation details in the feature space adds to the complexity of the problem. Over years several techniques have been proposed based on object oriented technique and pattern detection.
\end{abstract}

This proposed work introduces a novel rotation invariant technique for identifying number associated with contours taking into consideration friend pattern chain of the significant pixels representing a digit in a number.

\section{KEYWORD \\ Friend pattern chain, topological, elevation, DSM, DEM.}

\section{INTRODUCTION}

Topological map hosts different types of terrain information that plays a crucial role in inferential studies related to morphological features. The information includes point feature such as land marks, line feature such as rivers and contours and polygon features such as contour enclosures and lakes. With every identifiable morphological feature, additional information such as attributes is explicitly associated. Additional information includes elevation for contour, names for land marks, names for rivers etc. to name a few. Information's pertaining to features is used in various inferential studies related to the terrain analysis. These morphological features in a topological sheet are uniquely identified by different color code. These color code assists in visual interpretation of the features.

One such important information is contour and its elevation. Contour and its elevation are usually represented using brown color code. Elevation detail is associated either with each contour or at certain intervals. Contour and its elevation are used in order to create Digital Elevation Model and Digital Surface Model.

Current techniques for text detection are capable of identifying the various text features associated with an image but are highly dependent on the orientation or positioning of the text. In addition to this assistance of additional algorithms are required (such as edge detection for horizontal and vertical strokes) in turn resulting in substantial increase in the complexity of the identification process.

In order to extract contour and its elevation, firstly the topographic sheet has to be color segmented. Color segmentation process is used in order to highlight only contour and its elevation and subside other features. Then the segmented feature under goes through two sub processes, contour identification and elevation detail identification.

This work tries to introduce a novel pattern recognition trail called the friend pattern chain. Friend Pattern Chain can be defined as a chain of values that keeps in account the repetition of similar types of friend occurrences in a dataset representing an object.

This proposed work aim at designing and developing an effective and efficient algorithm for automatically determining elevation related with contour that tries to overcome rotation invariant problem by

- $\quad$ first acquiring the knowledge of the feature set by generating friend pattern chain

- determining frequencies

- $\quad$ associating priorities with frequency value based on uniqueness

- determination of reduced search space of comparing element

- $\quad$ identifying possible match for the detected feature

in this proposed work implementation is limited identification of elevation detail. 


\section{RELATED WORK}

Several research initiatives motivated towards identification of text from reference map use Optical Character Recognition $(\mathrm{OCR})$ technique as the primary basis for identifying test. This technique suffers from several inherent problems such as heavy reliance on quality of text. Yamamoto et al. [1] have proposed a method where numerals and symbols are recognized by the multi-angled parallelism (MAP) matching method, while small dots and lines are extracted by the MAP operation method. These results are then used to determine the value, position, and attributes of the elevations marked on the topographic maps. Multi Angled Parallelism (MAP) provides an efficient tool to detect miscellaneous linear features. However, parts of lines that pass through characters are often misclassified. It is proposed in [2], an improvement over MAP to automatically extract complete line networks with arbitrary orientation and curvature even when they pass through characters with minimal impact on the text content. The resulting text can then be processed for text grouping, reorientation, and recognition. Pezeshk et al. [3] have proposed automatic extraction of text based on new line representation technique and a set of directional morphological operations that are based on the MAP algorithm. A custom OCR is then used to recognize the extracted street labels and major place names. Pouderoux et al. [4] have proposed an automatic approach to extract and recognize toponyms based on image segmentation and connected component processing. Detected text area is then fed to OCR software for recognition. Luyang et al. [5] have used connected components of black layer extracted from topo-sheets to separate line Art, text, and icons. Anegawa et al. [6] have proposed a system for recognizing numeric strings from topographical maps, which is composed of extracting uncertain numeric strings using automatic recognition stage based on topographical map features only and corrected by the interactive recognition stage. Nakamura et al. [7] have described a method for recognizing character strings from topographical maps which consists of a bottom-up process for extracting character candidates from a map and a topdown process in which these character candidates are grouped into strings using linguistic knowledge of strings.

\section{METHODOLOGY}

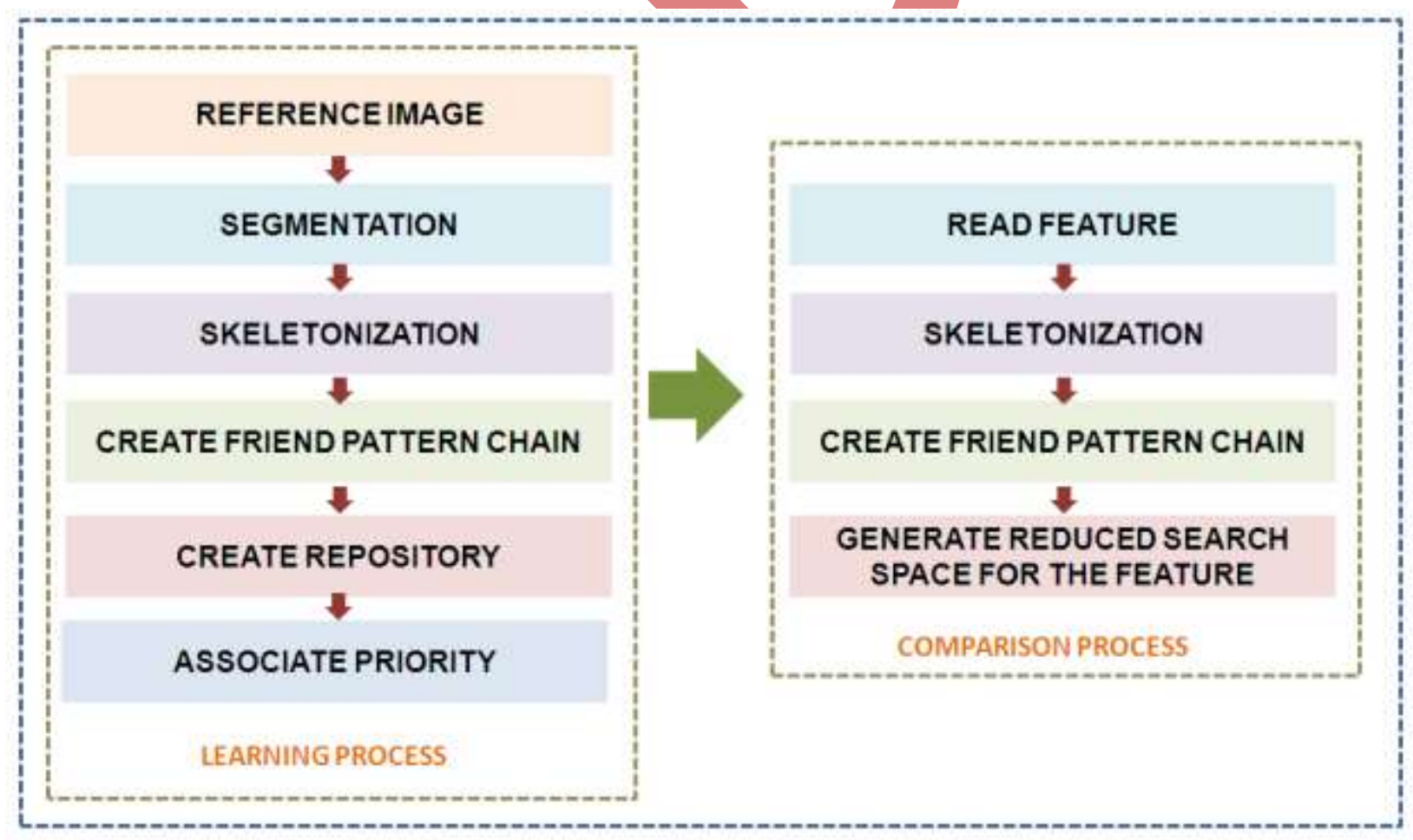

fig 1:- Schema diagram for proposed work.

In order to reduce the complexity of data processing in colored contour map, the reference map is converted to gray scale using the standard RGB to gray (i.e weighted sum of Red, Green and Blue component) conversion scheme $G(x, y)=0.2989 * R(x, y)+0.5870 * G(x, y)+0.1140 * B(x, y)$

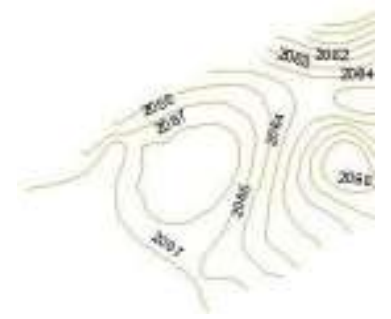

(a)

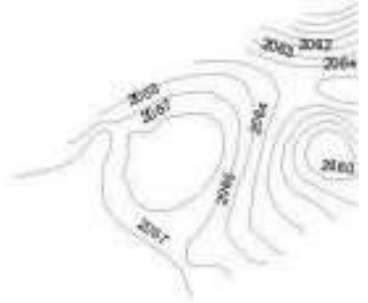

(b) 
Fig 2:- (a) color reference map (b) gray reference map

The proposed work has been organized into following identifiable modules.

\section{Segmentation of contour and elevation}

The contour map pertaining to a terrain consists of several feature, of the set of features most important feature are contour and its elevation. In a contour map these features are represent using different color code in order to make it more presentable. This color code provides the basis for segmenting contours and elevation details. Here in this work a simple multi level thresholding technique was used to separate the contours from the elevation detail. The digital number range taken for text is 0 to 50 and for contour is 145 to 198 the result obtained was as follows

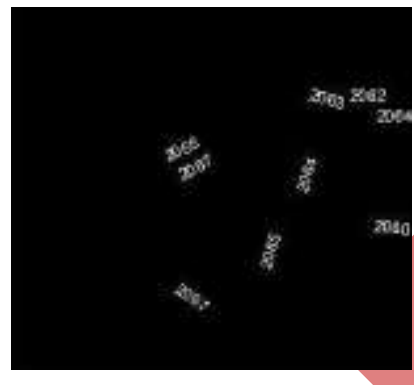

(a)

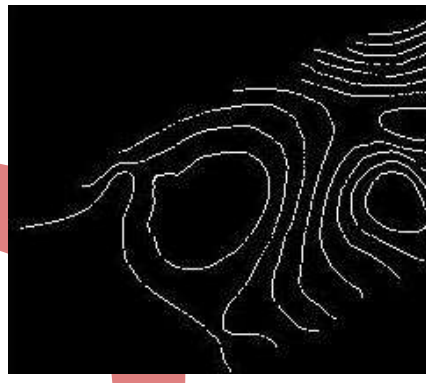

(b)

Fig 3:- (a) Segmented elevation detail (b) Segmented contour detail

\section{Application of morphological operator for creating single pixel representation of features}

A feature of interest in the reference map may be represented using single pixel width or multiple pixel width. If an object of interest is represented using multiple pixel width then for the ease of operation thinning morphological operator can be applied to represent the object using single pixel width.

\section{Repository creation for digits encountered in segmented image}

For identifying the digits extracted from segmented image as any one of the significant digit from 0 to 9 , firstly we need to create repository for the same. This repository host friend pattern chain for each and every identified character. The friend pattern chain matches closely with the chain code scheme but in this case rather than creating sequence we store the frequency of occurrence of pixel with same friend pattern. In a window of $3 \times 3$ following are the location where friends may be encountered, let us consider $\mathrm{g}(\mathrm{i}, \mathrm{j})$ be the coordinates for which we are determining friends

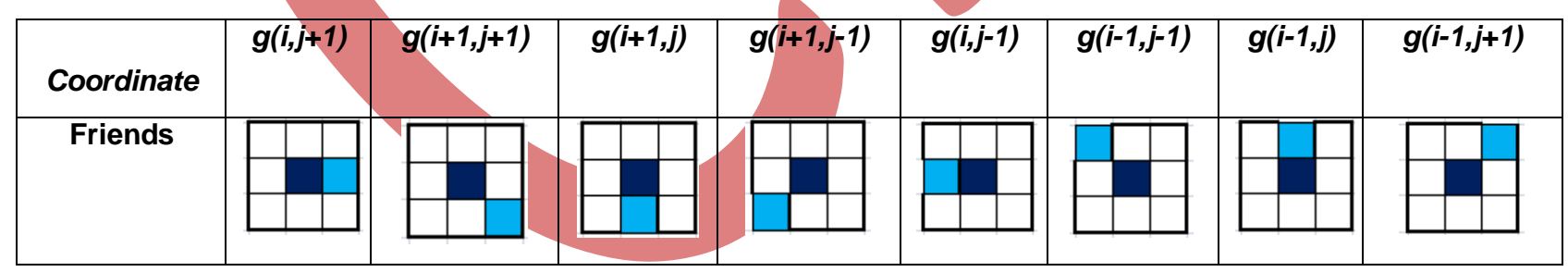

Following cases identifies the types of friends a pixel may have.

\begin{tabular}{|c|c|c|c|c|c|}
\hline Coordinates & 0 friends & 1 friend & 2 friend & 3 friend & 4 friend \\
\hline Friends & & & & & \\
\hline & & & & & \\
\hline
\end{tabular}

The position of friends might be any of the eight neighbors.

\section{Creation of friend pattern chain}

Consider the following sample case for creating friend pattern chain of a character identified

\begin{tabular}{|l|l|l|l|}
\hline Image & $\begin{array}{l}\text { Binary } \\
\text { Pattern }\end{array}$ & Friend Pattern & friend pattern chain \\
\hline
\end{tabular}




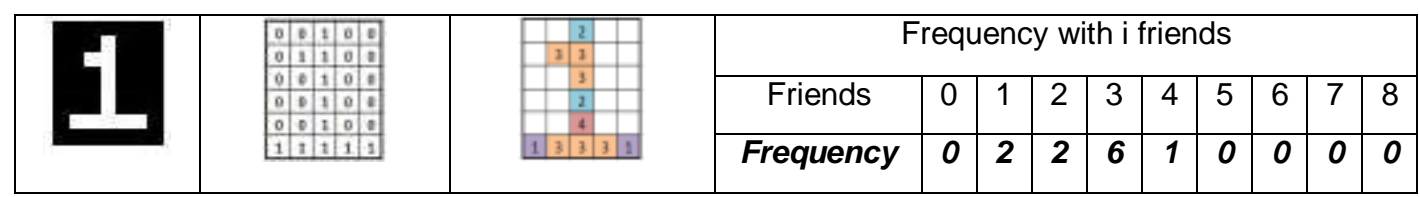

The friend pattern chain for the identifed character is 022610000 , so now instead of maintaining the coordinate pattern for the character we can create such friend pattern chain and take this into account for idetifying digits in a text.

So like wise friend pattern chain is to be created for all the digit from 0 to 9 .

Table 1. Database Created for the text along with friend pattern chain

\begin{tabular}{|c|c|c|c|c|c|c|c|c|c|c|c|c|c|c|}
\hline Image & Boundary & Thres & $\begin{array}{c}\text { Thinned } \\
\text { Image }\end{array}$ & $\begin{array}{l}\text { Friend } \\
\text { Pattern }\end{array}$ & & \multicolumn{9}{|c|}{ Friend Pattern Chain } \\
\hline \multirow{2}{*}{0} & & & & & Friends & 0 & 1 & 2 & 3 & 4 & 5 & 6 & 7 & 8 \\
\hline & & & & & Frequency & 0 & 0 & 41 & 0 & 0 & 0 & 0 & 0 & 0 \\
\hline \multirow{2}{*}{1} & & & & & Friends & 0 & 1 & 2 & 3 & 4 & 5 & 6 & 7 & 8 \\
\hline & & & & & Frequency & 0 & 4 & 23 & 3 & 0 & 0 & 0 & 0 & 0 \\
\hline \multirow[t]{2}{*}{2} & & & & & Friends & 0 & 1 & 2 & 3 & 4 & 5 & 6 & 7 & 8 \\
\hline & & & & & Frequency & 0 & 2 & 24 & 0 & 0 & 0 & 0 & 0 & 0 \\
\hline \multirow{2}{*}{3} & & & & & Friends & 0 & 1 & 2 & 3 & 4 & 5 & 6 & 7 & 8 \\
\hline & & & & & Frequency & 0 & 3 & 26 & 2 & 0 & 0 & 0 & 0 & 0 \\
\hline \multirow[t]{2}{*}{4} & & & & & Friends & 0 & 1 & 2 & 3 & 4 & 5 & 6 & 7 & 8 \\
\hline & & & & & Frequency & 0 & 1 & 31 & 3 & 0 & 0 & 0 & 0 & 0 \\
\hline \multirow{2}{*}{5} & & & & & Friends & 0 & 1 & 2 & 3 & 4 & 5 & 6 & 7 & 8 \\
\hline & & & & & Frequency & 0 & 2 & 30 & 0 & 0 & 0 & 0 & 0 & 0 \\
\hline \multirow{2}{*}{6} & & & & & Friends & 0 & 1 & 2 & 3 & 4 & 5 & 6 & 7 & 8 \\
\hline & & & & & Frequency & 0 & 1 & 38 & 1 & 0 & 0 & 0 & 0 & 0 \\
\hline \multirow{2}{*}{7} & & & & & Friends & 0 & 1 & 2 & 3 & 4 & 5 & 6 & 7 & 8 \\
\hline & & & & & Frequency & 0 & 2 & 21 & 0 & 0 & 0 & 0 & 0 & 0 \\
\hline \multirow{2}{*}{8} & & & & & Friends & 0 & 1 & 2 & 3 & 4 & 5 & 6 & 7 & 8 \\
\hline & & & & & Frequency & 0 & 0 & 41 & 2 & 0 & 0 & 0 & 0 & 0 \\
\hline \multirow[t]{2}{*}{9} & & & & & Friends & 0 & 1 & 2 & 3 & 4 & 5 & 6 & 7 & 8 \\
\hline & & & & & Frequency & 0 & 1 & 36 & 3 & 0 & 0 & 0 & 0 & 0 \\
\hline
\end{tabular}

So, now the summarized representation for the friend pattern chain would be,

\begin{tabular}{|c|c|c|c|c|c|c|c|c|c|c|}
\hline \multirow{2}{*}{$\begin{array}{c}\text { Digits } \\
\text { Definition }\end{array}$} & \multicolumn{7}{|c|}{ Friend Patten Chain (Friends) } & Total Pixel \\
\cline { 2 - 10 } & 0 & 1 & 2 & 3 & 4 & 5 & 6 & 7 & 8 & Sum of all \\
\hline 0 & 0 & 0 & 41 & 0 & 0 & 0 & 0 & 0 & 0 & 41 \\
\hline 1 & 0 & 4 & 23 & 3 & 0 & 0 & 0 & 0 & 0 & 30 \\
\hline 2 & 0 & 2 & 24 & 0 & 0 & 0 & 0 & 0 & 0 & 26 \\
\hline 3 & 0 & 3 & 26 & 2 & 0 & 0 & 0 & 0 & 0 & 31 \\
\hline 4 & 0 & 1 & 31 & 3 & 0 & 0 & 0 & 0 & 0 & 35 \\
\hline 5 & 0 & 2 & 30 & 0 & 0 & 0 & 0 & 0 & 0 & 32 \\
\hline
\end{tabular}




\begin{tabular}{|l|l|l|l|l|l|l|l|l|l|l|}
\hline 6 & 0 & 1 & 38 & 1 & 0 & 0 & 0 & 0 & 0 & 40 \\
\hline 7 & 0 & 2 & 21 & 0 & 0 & 0 & 0 & 0 & 0 & 23 \\
\hline 8 & 0 & 0 & 41 & 2 & 0 & 0 & 0 & 0 & 0 & 43 \\
\hline 9 & 0 & 1 & 36 & 3 & 0 & 0 & 0 & 0 & 0 & 40 \\
\hline
\end{tabular}

\section{Significance of having pixel with 0 or no friends}

Although the images representing numbers to be recognized often is considered to hold only the significant values. In reality these reference images representing the numbers may be infested with micro errors known errors referred to as salt and pepper noise. Salt and pepper noise are errors that are randomly distributed over the image. Salt and pepper implicitly refer white and black pixel that does not form part of any if the identifiable image.

Salt and pepper noise are morphologically categorized by an object having single pixel width, so when a pixel is encountered with 0 friends it can be automatically classified as salt or pepper noise.

\section{Significance for total pixel count}

If there are errors in the reference images that cannot be categorized as salt and pepper noise then assuming that an object of interest has some significant pixel count, a threshold value can be set to identify an object as a significant object.

\section{Association of priority with the friends in the pattern}

Upon determining the friend chain pattern for identified object, it becomes essential to associate weights with each and every frequency in the friend pattern chain, the purpose of associating priority or weight is to highlight or emphasize on key features that a pattern should have in order to qualify it as a particular symbol. As in the sample case discussed above the frequency pattern pertaining to 1 has to have; 2 coordinates with 2 friends, 1 coordinate with 4 friends and 6 coordinates with 3 friends, so in order to qualify the extracted symbol as 1 it should at least satisfy this criteria.

Upon analyzing the friend pattern chains it is found that some frequency values are same at a friend value, some frequency values are same as well as different for instances and where as some are uniquely different, so priority values should be assigned in a manner that the frequency with maximum distribution should be assigned first priority where as frequency with least distributing should be assigned least priority where as other can be assigned relative priority.

To determine the frequency that needs to be assigned highest and subsequent priorities we need to see the distribution of the frequency values.

\begin{tabular}{|c|c|c|c|c|c|c|c|c|c|c|c|c|c|c|c|c|c|c|}
\hline \multirow{2}{*}{ Frequency } & \multicolumn{2}{|c|}{0} & \multicolumn{2}{|c|}{1} & \multicolumn{2}{|c|}{2} & \multicolumn{2}{|c|}{3} & \multicolumn{2}{|c|}{4} & \multicolumn{2}{|r|}{5} & \multicolumn{2}{|c|}{6} & \multicolumn{2}{|c|}{7} & \multicolumn{2}{|c|}{8} \\
\hline & 0 & 10 & 0 & 2 & 21 & 1 & 0 & 4 & 0 & 10 & 0 & 10 & 0 & 10 & 0 & 10 & 0 & 10 \\
\hline \multirow{8}{*}{ 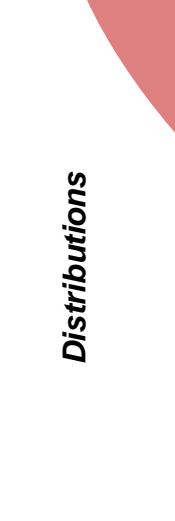 } & $x$ & $\mathbf{x}$ & 1 & 3 & 23 & 1 & 1 & 1 & $\mathbf{x}$ & $\mathbf{x}$ & $x$ & $\mathbf{x}$ & $\mathbf{x}$ & $\mathbf{x}$ & $\mathbf{x}$ & $\mathbf{x}$ & $\mathbf{x}$ & $\mathbf{x}$ \\
\hline & $\mathbf{x}$ & $x$ & 2 & 3 & 24 & 1 & 2 & 2 & $x$ & $\mathbf{x}$ & $\mathbf{x}$ & $\mathbf{X}$ & $\mathbf{x}$ & $\mathbf{x}$ & $\mathbf{x}$ & $\mathbf{x}$ & $\mathbf{x}$ & $\mathbf{x}$ \\
\hline & $\mathbf{x}$ & $\mathbf{x}$ & 3 & 1 & 26 & 1 & 3 & 3 & $\mathbf{x}$ & $\mathbf{x}$ & $\mathbf{x}$ & $\mathbf{x}$ & $\mathbf{x}$ & $\mathbf{x}$ & $\mathbf{x}$ & $\mathbf{x}$ & $\mathbf{x}$ & $\mathbf{x}$ \\
\hline & $\mathbf{x}$ & $x$ & 4 & 1 & 30 & 1 & $\bar{x}$ & $x$ & $\mathbf{x}$ & $\mathbf{x}$ & $\mathbf{x}$ & $\mathbf{x}$ & $\mathbf{x}$ & $\mathbf{x}$ & $\mathbf{x}$ & $\mathbf{x}$ & $\mathbf{x}$ & $\mathbf{x}$ \\
\hline & $\mathbf{x}$ & $\mathbf{x}$ & $\mathbf{x}$ & $x$ & 31 & 1 & $x$ & $\mathbf{x}$ & $\mathbf{x}$ & $x$ & $\mathbf{x}$ & $\mathbf{x}$ & $\mathbf{x}$ & $\mathbf{x}$ & $\mathbf{x}$ & $\mathbf{x}$ & $\mathbf{x}$ & $\mathbf{x}$ \\
\hline & $\mathbf{x}$ & $\mathbf{x}$ & $\mathbf{x}$ & $\mathbf{x}$ & 36 & 1 & $\mathbf{x}$ & $\mathbf{x}$ & $\mathbf{x}$ & $\mathbf{x}$ & $\mathbf{x}$ & $\mathbf{x}$ & $\mathbf{x}$ & $\mathbf{x}$ & $\mathbf{x}$ & $\mathbf{x}$ & $\mathbf{x}$ & $\mathbf{x}$ \\
\hline & $\mathbf{x}$ & $\mathbf{x}$ & $\mathbf{x}$ & $\mathbf{x}$ & 38 & 1 & $\mathbf{x}$ & $\mathbf{x}$ & $\mathbf{x}$ & $\mathbf{x}$ & $\mathbf{x}$ & $\mathbf{x}$ & $\mathbf{X}$ & $\mathbf{x}$ & $\mathbf{x}$ & $\mathbf{x}$ & $\mathbf{x}$ & $\mathbf{x}$ \\
\hline & $\mathbf{x}$ & $\mathbf{x}$ & $\mathbf{x}$ & $\mathbf{x}$ & 41 & 2 & $\mathbf{x}$ & $\mathbf{x}$ & $\mathbf{x}$ & $\mathbf{x}$ & $\mathbf{x}$ & $\mathbf{x}$ & $\mathbf{x}$ & $\mathbf{x}$ & $\mathbf{x}$ & $\mathbf{x}$ & $\bar{x}$ & $\mathbf{x}$ \\
\hline
\end{tabular}

The above table gives the distributing of unique values present in the friend pattern chain, frequency 2 has the highest number of unique values followed by 1 and then followed by 3 rest of the frequency values has only 1 unique value. The above can be explained by creating the graph of dissimilar values. 


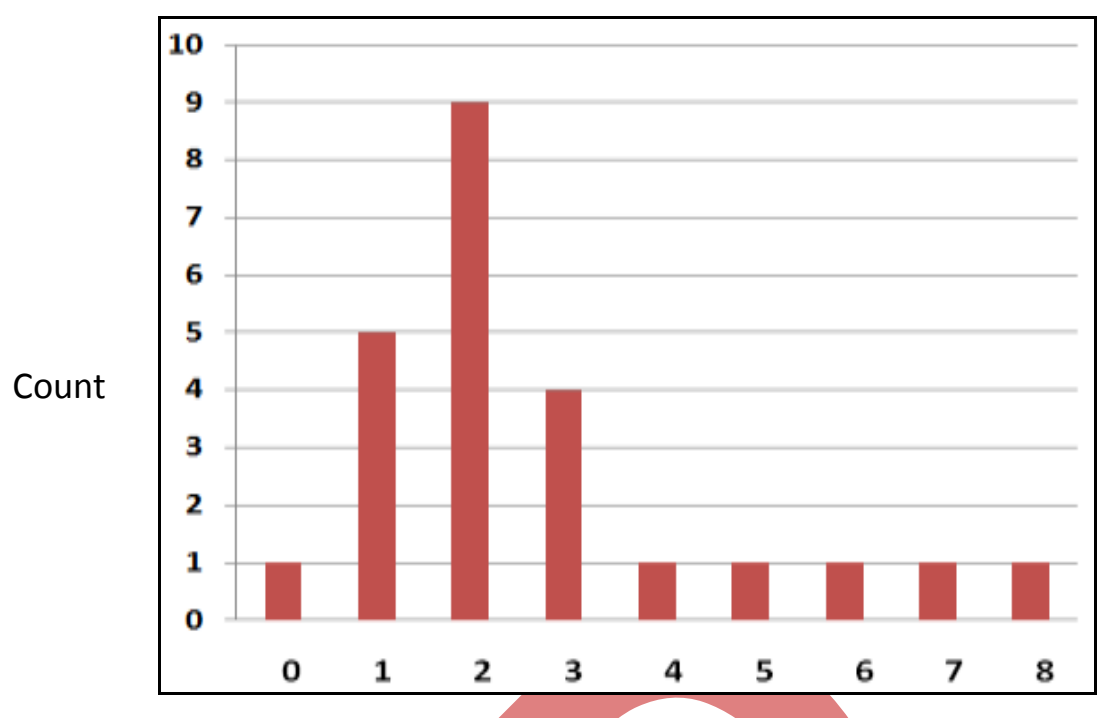

No. of unique values for frequency $i$

\begin{tabular}{|c|c|c|c|c|c|c|c|c|c|c|c|c|c|c|c|c|c|c|}
\hline Frequency & \multicolumn{2}{|c|}{0} & \multicolumn{2}{|c|}{1} & \multicolumn{2}{|c|}{2} & \multicolumn{2}{|c|}{3} & \multicolumn{2}{|c|}{4} & \multicolumn{2}{|c|}{5} & \multicolumn{2}{|r|}{6} & \multicolumn{2}{|c|}{7} & \multicolumn{2}{|c|}{8} \\
\hline \multirow{9}{*}{ 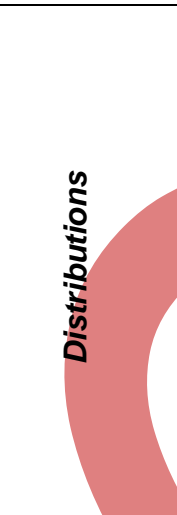 } & 0 & 10 & 0 & 2 & 21 & 1 & 0 & 4 & 0 & 10 & 0 & 10 & 0 & 10 & 0 & 10 & 0 & 10 \\
\hline & $\mathbf{x}$ & $\mathbf{x}$ & 1 & 3 & 23 & 1 & 1 & 1 & $\mathbf{x}$ & $\bar{x}$ & $x$ & $\mathbf{x}$ & $\mathbf{x}$ & $\bar{x}$ & $x$ & $x$ & $\mathbf{x}$ & $\mathbf{x}$ \\
\hline & $\mathbf{x}$ & $\mathbf{X}$ & 2 & 3 & 24 & 1 & 2 & 2 & $x$ & $x$ & $x$ & $x$ & $\mathbf{X}$ & $\bar{x}$ & $x$ & $x$ & $x$ & $\mathbf{x}$ \\
\hline & $x$ & $x$ & 3 & 1 & 26 & 1 & 3 & 3 & $\mathbf{x}$ & $x$ & $x$ & $\mathbf{x}$ & $\mathbf{X}$ & $\mathbf{x}$ & $x$ & $x$ & $x$ & $\mathbf{x}$ \\
\hline & $\bar{x}$ & $\mathbf{x}$ & 4 & 1 & 30 & 1 & $x$ & $\mathbf{x}$ & $\mathbf{x}$ & $\mathbf{x}$ & $x$ & $x$ & $\mathbf{x}$ & $\mathbf{x}$ & $\mathbf{x}$ & $\mathbf{x}$ & $\mathbf{x}$ & $\mathbf{x}$ \\
\hline & $\mathbf{x}$ & $x$ & $\bar{x}$ & $x$ & 31 & 1 & $x$ & $x$ & $\mathbf{x}$ & $x$ & $x$ & $x$ & $x$ & $x$ & $x$ & $\mathbf{x}$ & $\mathbf{x}$ & $\mathbf{x}$ \\
\hline & $\mathbf{x}$ & $\mathbf{x}$ & $\mathbf{x}$ & $\mathbf{x}$ & 36 & 1 & $\mathbf{x}$ & $x$ & $x$ & $\mathbf{x}$ & $\mathbf{x}$ & $\mathbf{x}$ & $x$ & $x$ & $\mathbf{x}$ & $\mathbf{x}$ & $\mathbf{x}$ & $\mathbf{x}$ \\
\hline & $\mathbf{x}$ & $\mathbf{x}$ & $\bar{x}$ & $\mathbf{x}$ & 38 & 1 & $\mathbf{x}$ & $\mathbf{x}$ & $x$ & $x$ & $\mathbf{x}$ & $\mathbf{x}$ & $\mathbf{x}$ & $\mathbf{x}$ & $\mathbf{x}$ & $\mathbf{x}$ & $\mathbf{x}$ & $\mathbf{x}$ \\
\hline & $\mathbf{x}$ & $\mathbf{x}$ & $\mathbf{x}$ & $\mathbf{x}$ & 41 & 2 & $\mathbf{x}$ & $\mathbf{x}$ & $\mathbf{x}$ & $\bar{x}$ & $x$ & $\mathbf{x}$ & $\mathbf{x}$ & $\mathbf{x}$ & $\mathbf{x}$ & $\mathbf{x}$ & $\mathbf{x}$ & $\mathbf{x}$ \\
\hline$\overline{\text { Priority }}$ & & 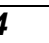 & & & & & & & & 4 & & 4 & & 4 & & 4 & & 4 \\
\hline
\end{tabular}

(Assumption Priority $1>2>3>4>\ldots$ )

On identification of the friend pattern chain for all possible digits, the friend pattern chains are stored in the repository. With the identified chain an identifier digit is associated along with priority values assigned with each frequency.

\section{Comparison of extracted pattern}

After the initial learning phase is over, the knowledge acquired can be used for identification of any digit that is encountered while navigating the reference data set.

While comparing the identified pattern with the reference map first the friend pattern chain of the identified digit is generated. The frequency value in the friend pattern chain is then compared with the values in the repository as per the priority assigned during learning phase. The friend pattern chain need not be compared with all the friend pattern chains in the repository instead the search space for the same can be reduced by selecting the frequency that matches the determined frequency in order of the priority.

\section{Test case 1:-}

\begin{tabular}{|c|c|c|c|c|c|c|c|c|c|c|c|c|}
\hline Image & Boundary & $\begin{array}{c}\text { Thinned } \\
\text { Image }\end{array}$ & \multicolumn{5}{|c|}{ Friend Pattern Chain } \\
\hline 7 & 7 & 3 & Friends & 0 & 1 & 2 & 3 & 4 & 5 & 6 & 7 & 8 \\
\cline { 5 - 13 } & & 7 & Frequency & 0 & 4 & 23 & 3 & 0 & 0 & 0 & 0 & 0 \\
\hline
\end{tabular}


Selection of search space from the repository (First Iteration)

\begin{tabular}{|c|c|c|c|c|c|c|c|c|c|c|}
\hline Digits & \multicolumn{9}{|c|}{ Friend Patten Chain (Friends) } & Total Pixel \\
\hline Definition & 0 & 1 & 2 & 3 & 4 & 5 & 6 & 7 & 8 & Sum of all \\
\hline 0 & 0 & 0 & 41 & 0 & 0 & 0 & 0 & 0 & 0 & 41 \\
\hline 1 & 0 & 4 & 23 & 3 & 0 & 0 & 0 & 0 & 0 & 30 \\
\hline 2 & 0 & 2 & 24 & 0 & 0 & 0 & 0 & 0 & 0 & 26 \\
\hline 3 & 0 & 3 & 26 & 2 & $\boldsymbol{0}$ & 0 & 0 & 0 & 0 & 31 \\
\hline 4 & 0 & 1 & 31 & 3 & 0 & 0 & 0 & 0 & 0 & 35 \\
\hline 5 & 0 & 2 & 30 & 0 & 0 & 0 & 0 & 0 & 0 & 32 \\
\hline 6 & 0 & 1 & 38 & 1 & 0 & 0 & 0 & 0 & 0 & 40 \\
\hline 7 & 0 & 2 & 21 & 0 & 0 & 0 & 0 & 0 & 0 & 23 \\
\hline 8 & 0 & 0 & 41 & 2 & 0 & 0 & 0 & 0 & 0 & 43 \\
\hline 9 & 0 & 1 & 36 & 3 & 0 & 0 & 0 & 0 & 0 & 40 \\
\hline Priority & 4 & 2 & 1 & 3 & 4 & 4 & 4 & 4 & 4 & \\
\hline
\end{tabular}

\begin{tabular}{|c|c|c|c|c|c|c|c|c|c|}
\hline \multirow{2}{*}{$\begin{array}{c}\text { Digits } \\
\text { Definition }\end{array}$} & \multicolumn{8}{|c|}{ Friend Patten Chain (Friends) } & \multirow{2}{*}{$\begin{array}{l}\text { Total Pixe } \\
\text { Sum of all }\end{array}$} \\
\hline & 0 & 1 & 3 & 4 & 5 & 6 & 7 & 8 & \\
\hline & 0 & 23 & 3 & 0 & 0 & 0 & 0 & 0 & 30 \\
\hline
\end{tabular}

Identified Digit is 1 .

Test case 2:-

\begin{tabular}{|c|c|c|c|c|c|c|c|c|c|c|c|c|}
\hline Image & Boundary & Thinned & & \multicolumn{8}{|c|}{ Friend Pattern Chain } & \\
\hline & & & Friends & 0 & 1 & 2 & 3 & 4 & 5 & 6 & 7 & 8 \\
\hline & & & Frequency & 0 & 0 & 41 & 0 & 0 & 0 & 0 & 0 & 0 \\
\hline
\end{tabular}

Selection of search space from the repository (First Iteration)

\begin{tabular}{|c|c|c|c|c|c|c|c|c|c|c|}
\hline Digits & \multicolumn{9}{|c|}{ Friend Patten Chain (Friends) } & Total Pixel \\
\hline Definition & 0 & 1 & 2 & 3 & 4 & 5 & 6 & 7 & 8 & Sum of all \\
\hline$\underline{\mathbf{0}}$ & 0 & 0 & 41 & 0 & 0 & 0 & 0 & 0 & 0 & 41 \\
\hline 1 & 0 & 4 & 23 & 3 & 0 & 0 & 0 & 0 & 0 & 30 \\
\hline 2 & 0 & 2 & 24 & 0 & 0 & 0 & 0 & 0 & 0 & 26 \\
\hline 3 & 0 & 3 & 26 & 2 & 0 & 0 & 0 & 0 & 0 & 31 \\
\hline 4 & 0 & 1 & 31 & 3 & 0 & 0 & 0 & 0 & 0 & 35 \\
\hline 5 & 0 & 2 & 30 & 0 & 0 & 0 & 0 & 0 & 0 & 32 \\
\hline 6 & 0 & 1 & 38 & 1 & 0 & 0 & 0 & 0 & 0 & 40 \\
\hline 7 & 0 & 2 & 21 & 0 & 0 & 0 & 0 & 0 & 0 & 23 \\
\hline 8 & 0 & 0 & 41 & 2 & 0 & 0 & 0 & 0 & 0 & 43 \\
\hline
\end{tabular}




\begin{tabular}{|c|c|c|c|c|c|c|c|c|c|c|}
\hline 9 & 0 & 1 & 36 & 3 & 0 & 0 & 0 & 0 & 0 & 40 \\
\hline Priority & 4 & 2 & 1 & 3 & 4 & 4 & 4 & 4 & 4 & \\
\hline
\end{tabular}

\begin{tabular}{|c|c|c|c|c|c|c|c|c|c|c|}
\hline Digits & \multicolumn{9}{|c|}{ Friend Patten Chain (Friends) } & Total Pixel \\
\hline Definition & 0 & 1 & 2 & 3 & 4 & 5 & 6 & 7 & 8 & Sum of all \\
\hline$\underline{0}$ & 0 & 0 & 41 & 0 & 0 & 0 & 0 & 0 & 0 & 41 \\
\hline 8 & 0 & 0 & 41 & 2 & 0 & 0 & 0 & 0 & 0 & 43 \\
\hline Priority & 4 & 2 & 1 & 3 & 4 & 4 & 4 & 4 & 4 & \\
\hline
\end{tabular}

Selection of search space from the repository (Second Iteration)

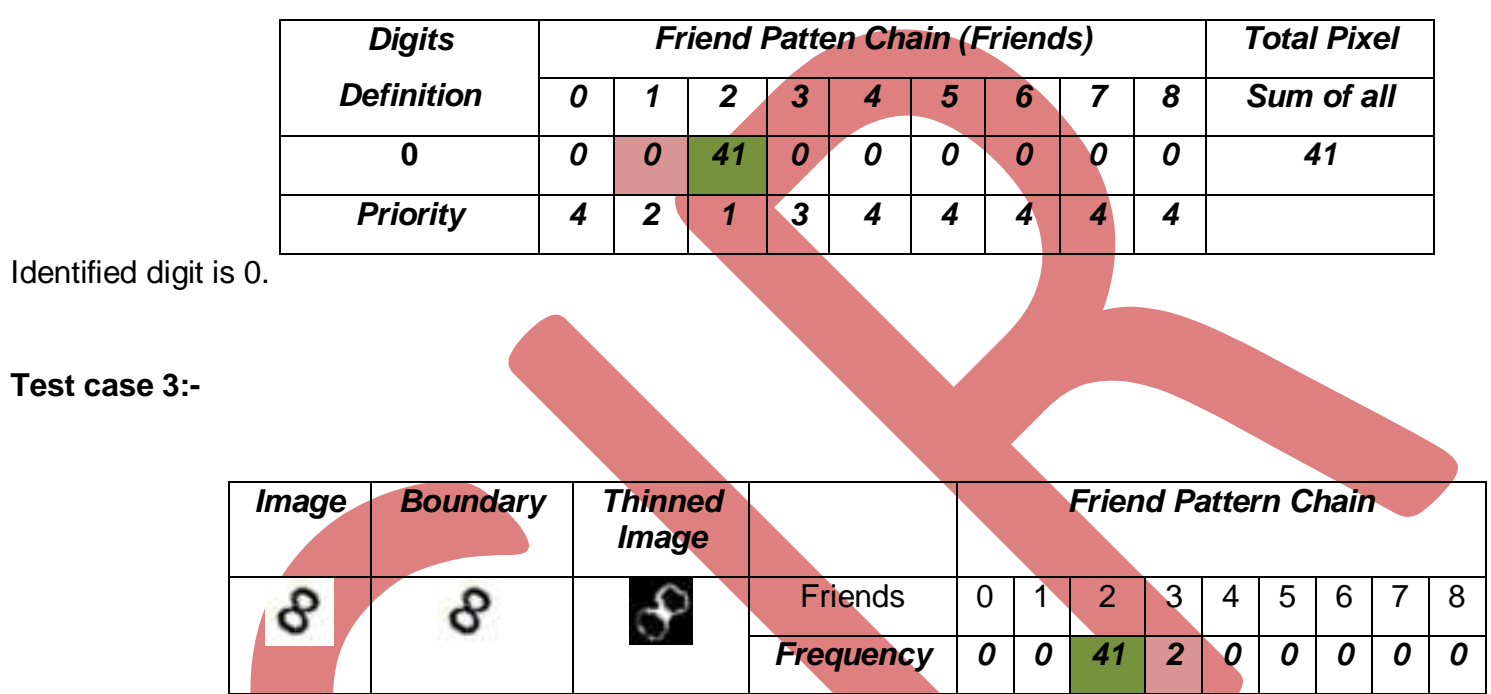

Selection of search space from the repository (First Iteration)

\begin{tabular}{|c|c|c|c|c|c|c|c|c|c|c|}
\hline \multirow{2}{*}{$\begin{array}{c}\text { Digits } \\
\text { Definition }\end{array}$} & 0 & 1 & 2 & 3 & 4 & 5 & 6 & 7 & 8 & Sum of all \\
\hline$\underline{0}$ & 0 & 0 & 41 & 0 & 0 & 0 & 0 & 0 & 0 & 41 \\
\hline 1 & 0 & 4 & 23 & 3 & 0 & 0 & 0 & 0 & 0 & 30 \\
\hline 2 & 0 & 2 & 24 & 0 & 0 & 0 & 0 & 0 & 0 & 26 \\
\hline 3 & 0 & 3 & 26 & 2 & 0 & 0 & 0 & 0 & 0 & 31 \\
\hline 4 & 0 & 1 & 31 & 3 & 0 & 0 & 0 & 0 & 0 & 35 \\
\hline 5 & 0 & 2 & 30 & 0 & 0 & 0 & 0 & 0 & 0 & 32 \\
\hline 6 & 0 & 1 & 38 & 1 & 0 & 0 & 0 & 0 & 0 & 40 \\
\hline 7 & 0 & 2 & 21 & 0 & 0 & 0 & 0 & 0 & 0 & 23 \\
\hline$\underline{8}$ & 0 & 0 & 41 & 2 & 0 & 0 & 0 & 0 & 0 & 43 \\
\hline 9 & 0 & 1 & 36 & 3 & 0 & 0 & 0 & 0 & 0 & 40 \\
\hline Priority & 4 & 2 & 1 & 3 & 4 & 4 & 4 & 4 & 4 & \\
\hline
\end{tabular}

\begin{tabular}{|c|c|c|c|c|c|c|c|c|c|c|}
\hline \multirow{2}{*}{$\begin{array}{c}\text { Digits } \\
\text { Definition }\end{array}$} & \multicolumn{7}{|c|}{ Friend Patten Chain (Friends) } & Total Pixel \\
\cline { 2 - 11 } & 0 & 1 & 2 & 3 & 4 & 5 & 6 & 7 & 8 & Sum of all \\
\hline 0 & 0 & 0 & 41 & 0 & 0 & 0 & 0 & 0 & 0 & 41 \\
\hline
\end{tabular}




\begin{tabular}{|c|c|c|c|c|c|c|c|c|c|c|}
\hline$\underline{8}$ & 0 & 0 & 41 & 2 & 0 & 0 & 0 & 0 & 0 & 43 \\
\hline Priority & 4 & 2 & 1 & 3 & 4 & 4 & 4 & 4 & 4 & \\
\hline
\end{tabular}

\section{Selection of search space from the repository (Second Iteration)}

\begin{tabular}{|c|c|c|c|c|c|c|c|c|c|c|}
\hline \multirow{2}{*}{$\begin{array}{c}\text { Digits } \\
\text { Definition }\end{array}$} & \multicolumn{7}{|c|}{ Friend Patten Chain (Friends) } & Total Pixel \\
\cline { 2 - 11 } & 0 & 1 & 2 & 3 & 4 & 5 & 6 & 7 & 8 & Sum of all \\
\hline$\underline{8}$ & 0 & 0 & 41 & 2 & 0 & 0 & 0 & 0 & 0 & 43 \\
\hline Priority & 4 & 2 & 1 & 3 & 4 & 4 & 4 & 4 & 4 & \\
\hline
\end{tabular}

Identified digit is 8 .

\section{CONCLUSION}

The implemented procedure is capable of identifying the number written in the same font and same text size to the degree of accuracy. The friend pattern representation comparatively takes less space as well as represents object in effective manner compared to that of traditional methods where entire pixel pattern is stored. The ability of determining reduced search space and association priority with the frequency values provides a best indication for initiation comparison adds to the capacity of the comparison process to determine best match in reduced time.

The work can be further refined to work with number written in the same font but different font size extended to generic fonts and generic font size.

\section{REFERENCES}

[1] Yamamoto, K., Yamada, H., Muraki, S., Symbol recognition and surface reconstruction from topographic map by parallel method, Proc. of the Second International Conference on Document Analysis and Recognition, 1993, pp 914917.

[2] Pezeshk, A., Improved Multi Angled Parallelism for separation of text from intersecting linear features in scanned topographic maps, proc. of IEEE International Conference on Acoustics Speech and Signal Processing (ICASSP), March 2010, pp. $1078-1081$.

[3] Pezeshk, A., Automatic Feature Extraction and Text Recognition From Scanned Topographic Maps, IEEE Transactions on Geoscience and Remote Sensing, Vol. 49, Issue: 12, Dec. 2011, pp. 5047 - 5063.

[4] Pouderoux J., Gonzato J., Pereira A., and Guitton P., Toponym Recognition in Scanned Color Topographic Maps, Proc. of International Conference on Document Analysis and Recognition (ICDAR), 2007, pp. 531-535.

[5] Luyang L, Nagy, G., Samal, A., Seth, S. , Yihong Xu, Cooperative text and line-art extraction from a topographic map, Proc. of the Fifth International Conference on Document Analysis and Recognition, 1999, PP. $467-470$.

[6] Anegawa, M., Shiku, O. , Nakamura, A. , Ohyamat, T. ; Kuroda, H., A system for recognizing numeric strings from topographical maps, proc. of the Third International Conference on Document Analysis and Recognition, 1995, pp. 940-943.

[7] Nakamura, A., Shiku, O. , Anegawa, M. , Nakamura, C. ; Kuroda, H., A method for recognizing character strings from maps using linguistic knowledge, Proc. of the Second International Conference on Document Analysis and Recognition, 1993., pp. $561-564$.

\section{Author' biography}

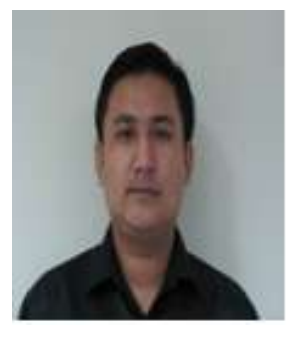

Mohan P Pradhan, He received B. Tech, M. Tech degrees in 2006 and 2009 respectively from Sikkim Manipal University, Sikkim, India. He is currently persuing his Ph. D for Sikkim Manipal University in the field of automatic digitization for GIS applications. He is currently working as Assistant Professor in department of Computer Science and Engineering, Sikkim Manipal Institute of Technology, Sikkim Manipal University since August 2006.

His research interest includes RS and GIS, Algorithms, Image Processing and 
Software Engineering.

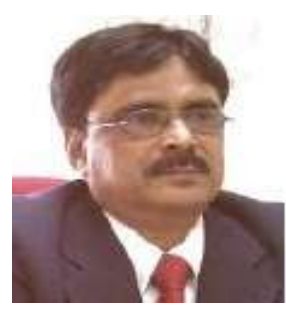

Professor (Dr.) M. K. Ghose is the Dean (R \& D), SMIT and Professor \& Head of the Department of Computer Science \& Engineering at Sikkim Manipal Institute of Technology, Mazitar, Sikkim, India since June, 2006. Prior to this, Dr. Ghose worked in the internationally reputed R \& D organization ISRO - during 1981 to 1994 at Vikram Sarabhai Space Centre, ISRO, Trivandrum in the areas of Mission Simulation and Quality \& Reliability Analysis of ISRO Launch vehicles and Satellite systems and during 1995 to 2006 at Regional Remote Sensing Service Centre, ISRO, IIT Campus, Kharagpur in the areas of RS \& GIS techniques for the natural resources management. He was also associated with Regional Engg. College (NIT), Silchar (1979 1981) as Teaching Asst. and Assam Central University, Silchar as COE and HOD of Computer Science Department (1997-2000).

His areas of research interest are Data Mining, Simulation \& Modelling, Network, Sensor Network, Information Security, Optimization \& Genetic Algorithm, Digital Image processing, Remote Sensing \& GIS and Software Engineering.

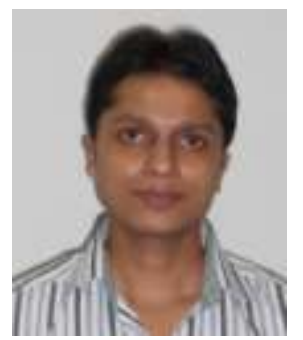

Gourav Mittal, he received his B. Tech in Computer Science from Sikkim Manipal University, Sikkim, India, 2012. He is currently working as Assistant Systems Engineer at Tata Consultancy Services.

His research interest includes Algorithms, Image Processing.

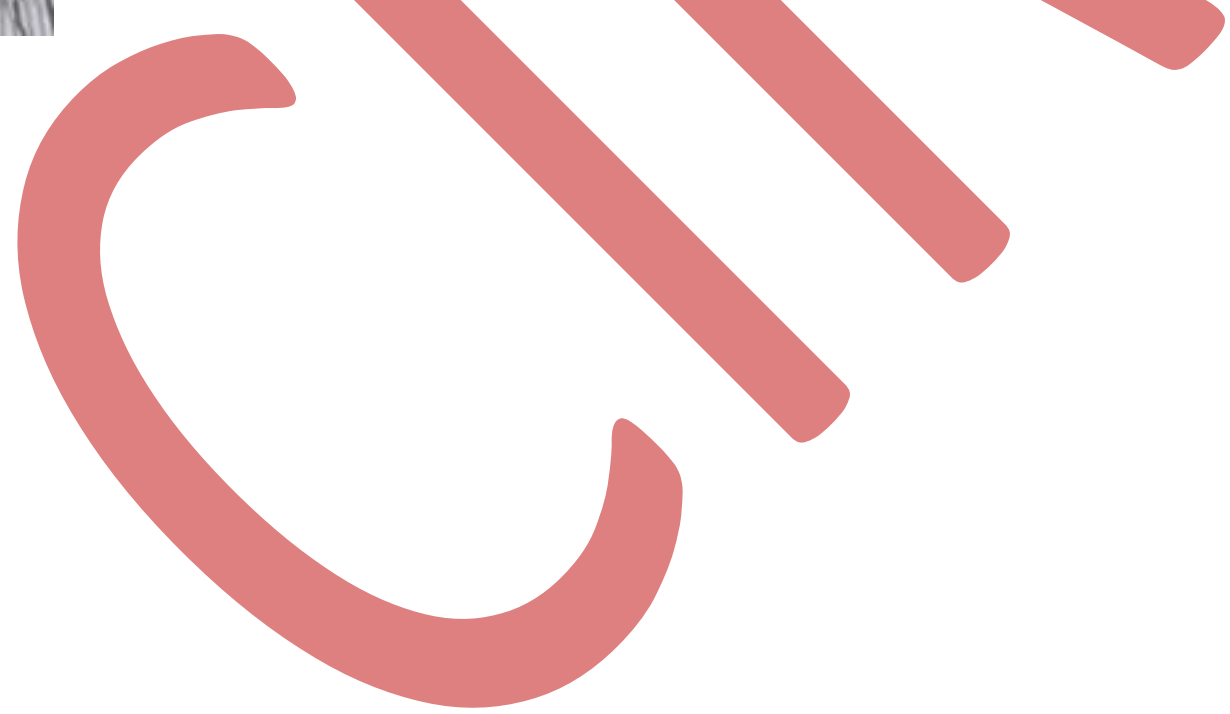

